\title{
Unprofessional behaviour on social media by medical students
}

\begin{abstract}
$\mathrm{S}$ ocial media are defined as a group of internet-based applications that allow the creation and exchange of user-generated content within a virtual community or network. ${ }^{1}$ Their rapid growth over the past decade has caused a paradigm shift in the way people communicate. The social media service Facebook reported 1.19 billion active users in 2013, and as early as 2008 the site was used by $64 \%$ of medical students, ${ }^{2}$ increasing to 93\% of Australian first-year health professional students in $2013,{ }^{3}$ suggesting that Facebook and other social media services form a growing part of students' lives.
\end{abstract}

Earlier research has examined the use of social media by medical students and doctors for personal purposes and discussed the implications for medical professionalism. ${ }^{4}$ One study $^{5}$ explored American medical schools' experience of medical students posting improper content online, including profanity, discriminatory language, depictions of alcohol intoxication, and sexually suggestive material. Such cases have led to disciplinary action and even expulsion. ${ }^{6}$ In response to the growing use of social media and concerns about its effect on professionalism, the Australian Medical Association published a guideline on online professionalism for medical students and practitioners in $2010 .^{7}$

Some behaviours are clearly unprofessional, such as the posting of patient information or any content that involves illegal activity. Depending on the context, depicting alcohol use may also be unprofessional.

We hypothesised that social media usage would be high among medical students, and that unprofessional material would be commonly posted and publicly available online. Additionally, we anticipated that medical students would know the relevant guidelines, ${ }^{7,8}$ and that they

\section{Abstract}

Objectives: To describe the social media usage patterns of medical students and to identify factors associated with their posting of unprofessional content on social media.

Design: Voluntary survey, delivered online.

Setting: All students in all 20 Australian medical schools were eligible to participate (16993 individuals).

Participants: Of 1027 initial respondents during the study period (29 March - 12 August 2013), 880 completed the survey.

Main outcome measures: Prevalence of unprofessional online behaviour on social media by medical students, as reported by students about their own and others' accounts.

Results: Posting of unprofessional content was self-reported by 306 students (34.7\%), mainly depictions of intoxication (301 students, 34.2\%) or illegal drug use (14 students, $1.6 \%$ ), or posting of patient information (14 students, 1.6\%). Posting of unprofessional content was associated with posting evidence of alcohol use and racist content online, MySpace use, and planning to change one's profile name after graduation. Factors associated with reduced unprofessional content included believing that videos depicting medical events with heavy alcohol use were inappropriate, and being happy with one's own social media portrayal. Exposure to guidelines on professional online conduct had no effect on posting behaviour.

Conclusions: Social media use was nearly universal in the surveyed cohort. Posting of unprofessional content was highly prevalent despite understanding that this might be considered inappropriate, and despite awareness of professionalism guidelines. Medical educators should consider approaches to this problem that involve more than simply providing guidelines or policies, and students should be regularly prompted to evaluate and moderate their own online behaviour.

would moderate their online behaviour accordingly.

The aim of our study was to assess social media use patterns among medical students, and to identify factors independently associated with reporting evidence of unprofessional behaviour on social media. We specifically investigated whether being exposed to guidelines on social media professionalism was associated with reduced prevalence of such behaviours.

\section{Methods}

An online survey comprising 35 questions with a skip-logic design was developed using the SurveyGizmo platform. Not every question was put to every respondent, as not all were applicable. The survey was conducted between 29 March and 12 August 2013.

Any student who was currently enrolled in a medical degree (undergraduate or postgraduate) at an Australian university was eligible to respond; 16993 students were potentially eligible, including international students. ${ }^{9}$ Students were recruited by contacting student organisations and Australian Medical Students Association (AMSA) representatives from each medical school and asking them to disseminate the recruitment information. The assistance of medical school deans was sought for internal promotion of the survey on online learning management systems (eg, Blackboard and Moodle). The study was also promoted by these groups through Facebook and Twitter. 
A standard consent page that detailed the purpose of the study was displayed before the survey was delivered. All responses were recorded, although only complete responses were included in the final analysis. As an incentive to complete the survey, students were offered the chance to win a prepaid debit card to the value of $\$ 500$, to be allocated randomly to one respondent; contact details were collected for this purpose in a separate form to ensure that identifying details could not be linked to the survey data. Vendorprovided duplicate protection technologies were used to prevent multiple responses by an individual. Funding was not received from any external body, with all costs borne by the authors of this article.

"Unprofessional content" was defined as an online depiction of illegal activity, overt intoxication or illicit drug use, or the posting of patient information. If students had removed unprofessional content posted by themselves or others on their account, it was not assessed as evidence of unprofessional behaviour.

Data were exported from the platform and analysed with SAS 9.4 (SAS Institute). Univariate comparisons were performed using $\chi^{2}$, Student $t$ - and Wilcoxon rank-sum tests as appropriate, according to the type and distribution of the data. Parametric data are reported as means and standard deviations, non-parametric data as medians with interquartile ranges (IQR), and categorical variables as numbers and percentages. Multivariable logistic regression of "unprofessional behaviour" was performed using backwards elimination techniques, with univariate $P<0.25$ as the cut-off for inclusion; results are reported as odds ratios (OR) with 95\% confidence intervals. Multicollinearity was assessed by evaluating coefficient changes between univariable and multivariable models and using variance inflation factors. $P<0.05$ (two-sided) was defined as statistically significant.

The study was approved as a lowrisk project by the Monash Health Research Ethics Committee (MUHREC LR 2012001495).

\section{Results}

It was not known how many of the 16993 students enrolled at Australian medical schools received notification about the survey, but 1027 from 20 medical schools (21 at the time of study enrolment) initially responded. Of these, 880 students fully completed the survey $(85.6 \%)$ and thus comprised the study cohort.

Of the 880 students who submitted complete responses, $534 \quad(60.6 \%)$ were undergraduates, 391 (44.4\%) were from Victorian medical schools, and 875 (99.4\%) used Facebook. The next most commonly used social networks were YouTube (853 students, $96.9 \%$ ) and blogging platforms (399 students, 45.3\%). Every student used at least one social network, with a mean of 5.5 networks per student (95\% CI, 5.31-5.67). Online professionalism teaching had been received by 305 students (34.9\%). Other demographic data are included in Box 1.

Unprofessional content was reported by 306 students as (34.7\%) being present on their accounts. Evidence of intoxication was reported by 301 students $(34.2 \%)$, evidence of illegal drug use by $14(1.6 \%)$, depiction of an illegal act by $10(1.1 \%)$, and the posting of patient information by 14 $(1.6 \%)$. The proportion of students who reported seeing unprofessional content on other medical students' accounts was much higher than that of those who reported it being present on their own account (Box 2).

Most students were aware of social media guidelines, with 475 (53.9\%) aware of a professional body guideline, $363(41.2 \%)$ of a university or clinical school guideline, and 584 (66\%; 95\% CI, 63\%-69\%) of at least one of the two. There was no association between knowledge of social media guidelines and unprofessional behaviour on social media (OR, 0.77; 95\% CI, 0.54-1.11; $P=0.16)$. Most respondents $(796,90.5 \%)$ agreed or strongly agreed with the statement that they were held to a higher standard of professionalism than the general community; 27 (3.1\%) disagreed and four $(0.5 \%)$ strongly disagreed (Box 3 and Box 4).
Of the 875 students who used Facebook, $848(96.9 \%)$ had changed the default security settings and 744 $(85.0 \%)$ had a private profile; 618 students $(70.6 \%)$ had increased their privacy and security settings by restricting content to groups or specific individuals.

After adjusting for covariates, unprofessional content was associated with students reporting that they had posted to their accounts evidence of alcohol use (OR, 6.50; 95\% CI, 4.42-9.56; $P<0.001)$ or racist content (OR, 2.45; 95\% CI, 1.15-5.20; $P=$ $0.02)$, that they had used MySpace (OR, 1.51; 95\% CI, 1.09-2.1; $P=0.01$ ), and planned to change their profile name after graduation (OR, 1.61; 95\% CI, 1.12-2.31; $P=0.01)$. Behaviours less likely to be associated with reporting of unprofessional content included believing that videos depicting medical events with heavy alcohol use are inappropriate (OR, 0.73; 95\% CI, 0.63-0.85; $P<0.001$ ), and being happy with their social media portrayal (OR, 0.57; 95\% CI, 0.45-0.74; $P<0.001$ ) (Box 4). Exposure to guidelines had no effect on students reporting unprofessional behaviours. The act of completing the survey itself caused 493 students $(56.0 \%)$ to check their privacy settings, and $307(34.9 \%)$ to change them.

\section{Discussion}

Our study found that social media use by the study population of medical students was nearly universal; further, $34.7 \%$ of respondents reported evidence of unprofessional content on their accounts. More students reported viewing unprofessional content on other students' accounts than on their own. Unprofessional content was reported despite exposure to guidelines and education about online professionalism.

Medical students are held to higher standards of professionalism than general university students, and we found that most students are aware of this. This is relevant, as unprofessional conduct (online or offline) by a student may lead to disciplinary action, and has also been found to be 
1 Demographic characteristics of students reporting or not reporting evidence of unprofessional behaviour*

\begin{tabular}{|c|c|c|c|c|}
\hline & All students & $\begin{array}{l}\text { Students reporting } \\
\text { no evidence } \\
\text { of unprofessional } \\
\text { behaviour }\end{array}$ & $\begin{array}{c}\text { Students reporting } \\
\text { evidence } \\
\text { of unprofessional } \\
\text { behaviour }\end{array}$ & $P$ \\
\hline Number & 880 & 574 & 306 & \\
\hline Median age (IQR), years & $22(20-24)$ [range, 16-40] & $22(20-24)$ & $22(21-24)$ & 0.95 \\
\hline \multicolumn{5}{|l|}{ Enrolment type } \\
\hline Undergraduate & $534(60.6 \%)$ & 359 (67.4\%) & $174(32.6 \%)$ & 0.11 \\
\hline Previous health care degree ${ }^{\dagger}$ & $98(28 \%)$ & $66(67 \%)$ & $32(33 \%)$ & 0.65 \\
\hline Domestic ${ }^{\ddagger}$ & $826(94.1 \%)$ & $527(63.8 \%)$ & $299(36.2 \%)$ & 0.001 \\
\hline \multicolumn{5}{|l|}{ Year of study } \\
\hline 1st & $184(20.9 \%)$ & $122(13.9 \%)$ & $62(7.0 \%)$ & 0.79 \\
\hline 2nd & $173(19.7 \%)$ & 117 (13.3\%) & $56(6.4 \%)$ & 0.48 \\
\hline $3 r d$ & $189(21.5 \%)$ & $124(14.1 \%)$ & $65(7.4 \%)$ & 0.93 \\
\hline 4th & $169(19.2 \%)$ & $103(11.7 \%)$ & $66(7.5 \%)$ & 0.21 \\
\hline 5th & $93(10.6 \%)$ & $55(6.3 \%)$ & $38(4.3 \%)$ & 0.21 \\
\hline 6th & $71(8.1 \%)$ & $53(6.0 \%)$ & $18(2.1 \%)$ & 0.09 \\
\hline \multicolumn{5}{|l|}{ University attended } \\
\hline Monash (Victoria) & $278(31.6 \%)$ & $190(21.6 \%)$ & $88(10.0 \%)$ & 0.19 \\
\hline Western Australia & $116(13.2 \%)$ & $73(8.3 \%)$ & $43(4.9 \%)$ & 0.60 \\
\hline Melbourne (Victoria) & $113(12.8 \%)$ & $79(8.9 \%)$ & $34(3.9 \%)$ & 0.29 \\
\hline Deakin (Victoria) & $78(8.9 \%)$ & $49(5.6 \%)$ & $29(3.3 \%)$ & 0.71 \\
\hline Queensland & $48(5.5 \%)$ & $30(3.4 \%)$ & $18(2.0 \%)$ & 0.75 \\
\hline New England (NSW) & $48(5.5 \%)$ & $25(2.8 \%)$ & $23(2.6 \%)$ & 0.06 \\
\hline Western Sydney (NSW) & $39(4.4 \%)$ & $29(3.3 \%)$ & $10(1.1 \%)$ & 0.30 \\
\hline Others & $160(18.1 \%)$ & 99 (61.9\%) & $61(38.1 \%)$ & 0.36 \\
\hline \multicolumn{5}{|c|}{ Received instruction about online professionalism } \\
\hline Yes & 305 (34.9\%) & $199(22.8 \%)$ & $106(12.1 \%)$ & 0.45 \\
\hline No & $421(48.2 \%)$ & $268(30.7 \%)$ & $153(17.5 \%)$ & \\
\hline Not sure & 147 (16.8\%) & 102 (11.7\%) & $45(5.2 \%)$ & \\
\hline
\end{tabular}

*All values reported as number (and column percentage) unless otherwise stated. $\dagger$ Percentage refers to medical postgraduates only. $\ddagger$ Australian and New Zealand citizens, and Australian permanent residents.

associated with lapses during later professional practice. ${ }^{6,10}$

\section{Factors associated with unprofessional content}

Several factors were associated with the presence of unprofessional content, including evidence of alcohol use or racist content, and planning to change one's profile name after graduation. Conversely, being happy with their social media portrayal appears to reduce the posting of unprofessional content by students. The implications of the association of unprofessional content with
MySpace use are uncertain, and may now be weaker, given the decline in use of this platform.

The association between posting unprofessional content and the intention to change social media profile names on graduation suggests that students were aware that they had unprofessional content on their accounts but were not intending to remove it. This is despite knowing about the relevant guidelines and believing they are subject to a higher expectation of professionalism than the general public. As changing one's profile name may not effectively conceal an individual's identity, such strategies provide a false sense of security and, paradoxically, encourage unprofessional behaviour. ${ }^{11}$

\section{Professionalism guidelines}

Medical associations and professional organisations have published guidelines and other literature ${ }^{7,8}$ in response to earlier research and reports in the media. Our results suggest that, despite the widespread dissemination and awareness of professional body guidelines in Australia, there appears to have been 
2 Unprofessional behaviours on medical students' social media accounts, self-reported (own account) $v$ observed (others' accounts)

A Self-reported v observed behaviours: general $\square$ Yes $\square$ No $\square$ Not sure

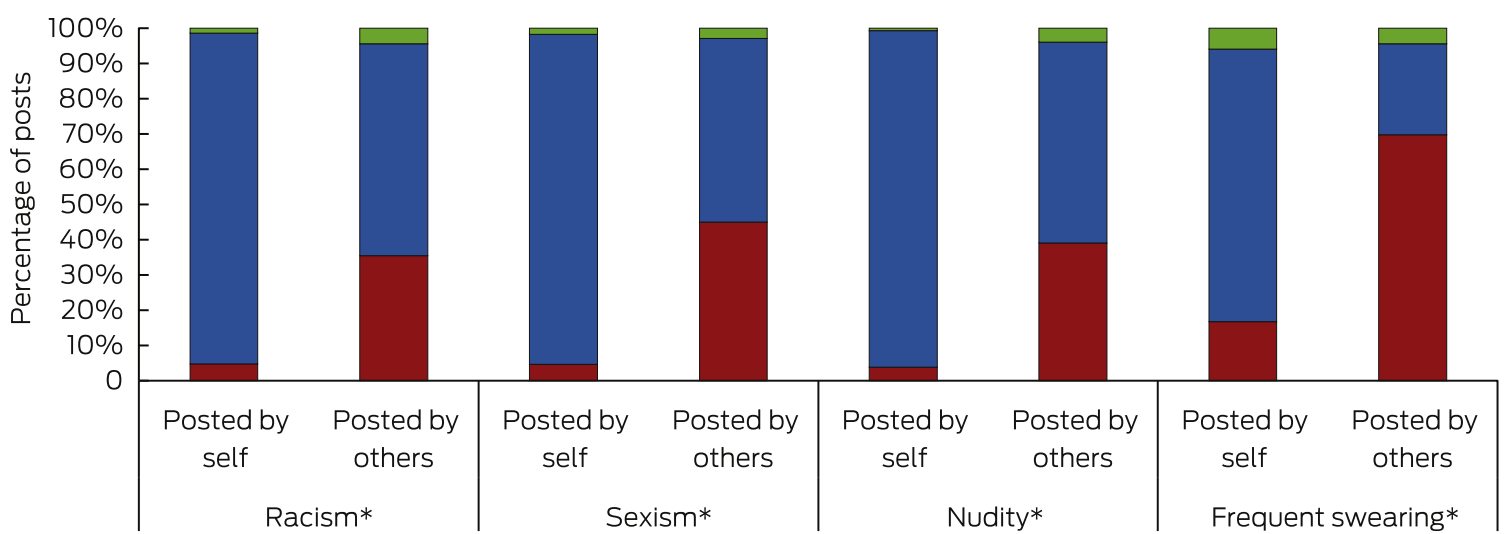

\section{B Self-reported v observed behaviours: institutions}

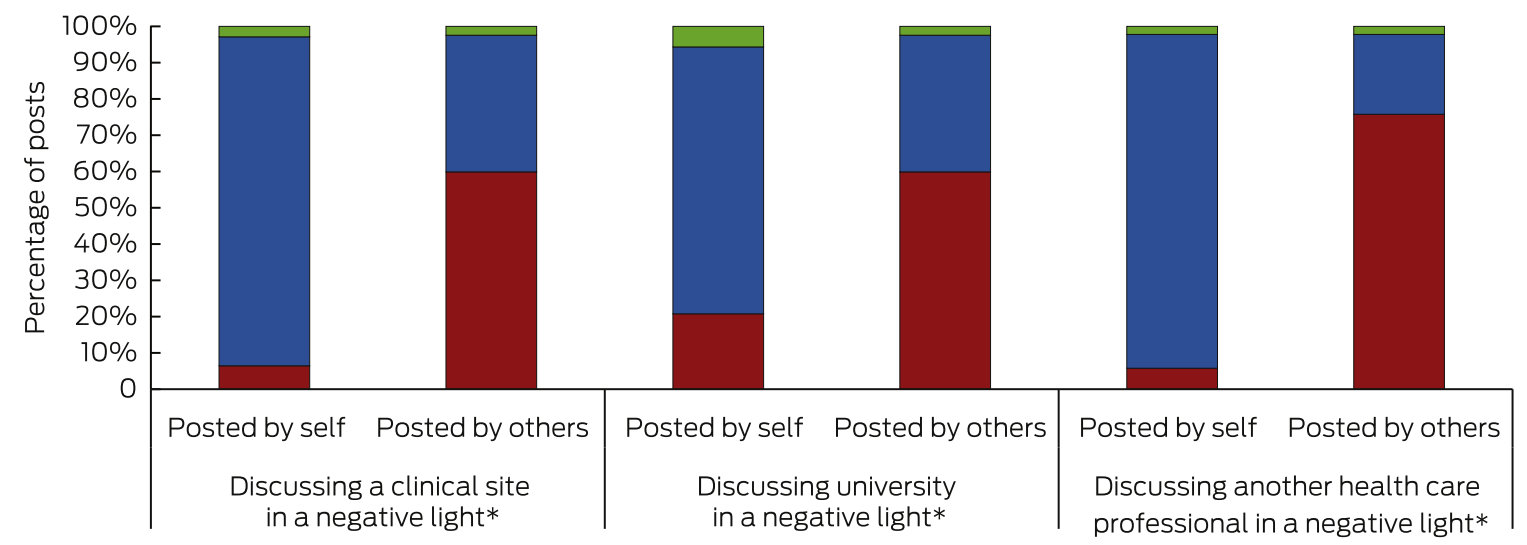

\section{Self-reported v observed unprofessional behaviours}

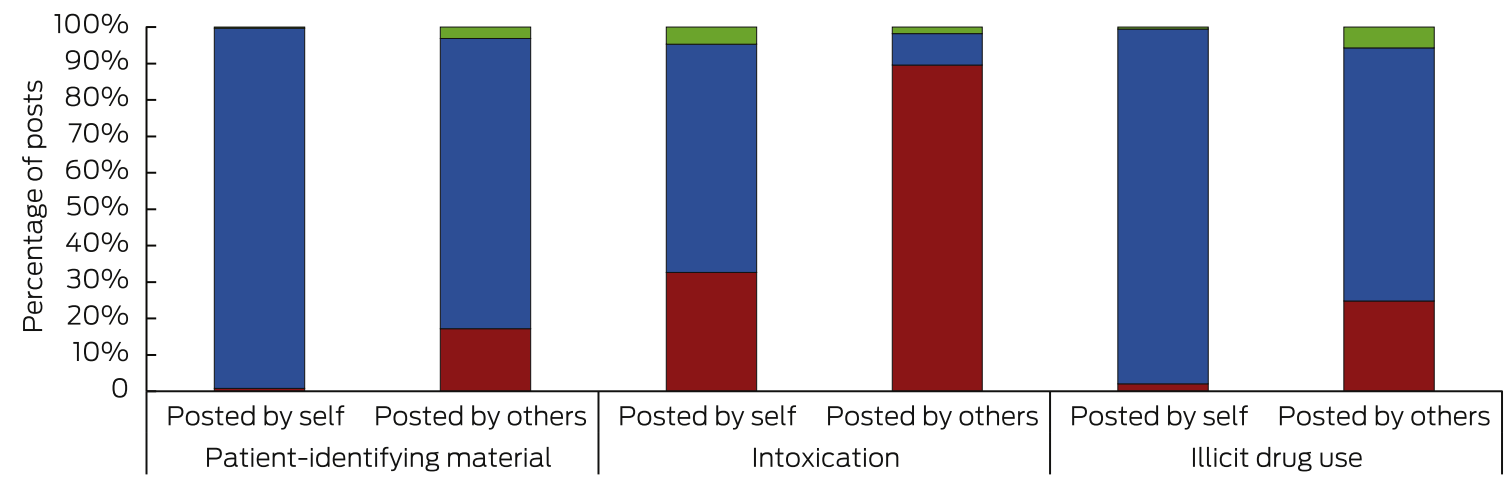

* Posted by self $\vee$ posted by others: $P<0.001$

only a minimal impact on medical students' behaviour.

There is a distinction between disseminating guidelines and formally integrating social media instruction into medical curricula. Senior clinicians and teachers who have not used social media may teach professionalism "largely in the context of the physician-patient relationship", 12 and be ill-equipped for teaching their juniors about professionalism in a social media context. ${ }^{13}$ Our findings show that reducing the unprofessional use of social media will require more than providing guidelines. 
3 Characteristics of students reporting or not reporting evidence of unprofessional behaviour* (univariate analysis)

Students reporting
no evidence of
unprofessional
behaviour

Do you use:

Facebook

Twitter

Reddit

Do you think it is appropriate to be Facebook "friends" with:

Allied health staff

Junior doctors

Nurses

Patients' families

Patients

Have you ever posted content which could be interpreted as:

Racist

Sexist

Containing frequent swearing

Discussing a clinical site in a negative light

What guides your professionalism on social media?

Concerns about appearing unprofessional to friends, family, or peers

Belief that as a medical student I am held to a higher standard of professionalism

University social media guidelines or policy guides my behaviour

Concern about appearing unprofessional to general public

Do you believe professionalism extends to social media presence?

Do you feel you have control over social media portrayal?

Are you happy with your social media portrayal?

Do you use a pseudonym for your profile name?

Do you plan to change your Facebook profile name after graduation?

Are you a domestic student?

$566 / 567(99.8 \%)$

208/574 (36.2\%)

$107 / 574(18.6 \%)$

$333 / 570(58.4 \%)$

443/571 (77.6\%)

$322 / 568(56.7 \%)$

$17 / 570$ (3.0\%)

$12 / 570(1.38 \%)$

$16 / 566(2.8 \%)$

$225 / 568(39.6 \%)$

$58 / 569(10.2 \%)$

$88 / 568(15.5 \%)$

407/574 (70.9\%)

$522 / 570(91.6 \%)$

$148 / 574(25.8 \%)$

414/574 (72.1\%)

$516 / 568$ (90.8\%)

$454 / 567(80.1 \%)$

$504 / 569$ (88.6\%)

46/566 (8.1\%)

$138 / 574(24.0 \%)$

$527 / 572(92.1 \%)$

$412 / 574(71.8 \%)$
Students reporting evidence of unprofessional behaviour

$304 / 305(99.6 \%)$

1.0

145/306 (47.4\%)

0.002

93/306 (30.4\%)

0.01

213/302 (70.5\%)

$<0.001$

$263 / 304(86.5 \%)$

0.0016

209/303 (70.0\%)

$<0.001$

7/303 (2.3\%)

0.67

3/303 (0.34\%)

0.33

25/302 (8.3\%)

$<0.001$

166/301 (55.2\%)

$<0.001$

91/301 (30.2\%)

$<0.001$

93/302 (30.8\%)

$<0.001$

192/306 (62.8\%)

0.01

274/304 (90.1\%)

0.53

58/306 (19.0\%)

0.02

198/306 (64.7\%)

0.03

246/306 (80.4\%)

0.0001

218/303 (71.9\%)

0.008

228/305 (74.7\%)

0.0001

40/306 (13.1\%)

0.02

$110 / 306(36.0 \%)$

0.0001

299/306 (97.7\%)

0.0008

219/306 (71.7\%) media guideline?

*All values reported as number and percentage of responders.

\section{Privacy settings}

The finding that $71 \%$ of students who used Facebook had set their account to "private" was higher than the $37.5 \%$ among US medical students reported in a 2008 study. $^{2}$ Private profiles allow a medical student to partition "personal" information from their public persona. However, they do not provide a completely safe sanctuary for unprofessional behaviour. Data leaks, changes to terms and conditions, and public dissemination of previously private information mean that private content posted to social media may still become more widely available.

Reading and interpreting online content is highly subjective, and the level of professionalism expected in 


\section{Reported factors independently associated with unprofessional behaviours (multivariate analysis)

\begin{tabular}{lcc} 
& OR (95\% Cl) & $P$ \\
\hline Evidence of any alcohol use (not intoxication) & $6.50(4.42-9.56)$ & $<0.0001$ \\
Evidence of posting racist content & $2.45(1.15-5.20)$ & 0.02 \\
MySpace use & $1.61(1.09-2.10)$ & 0.01 \\
Planning to change profile name upon graduation & $1.61(1.12-2.31)$ & 0.01 \\
$\begin{array}{l}\text { Believing that recording videos of medical events } \\
\text { depicting heavy alcohol use are inappropriate }\end{array}$ & $0.73(0.63-0.85)$ & $<0.0001$ \\
$\begin{array}{l}\text { Happy with social media portrayal } \\
\text { Read or been educated on social media guidelines }\end{array}$ & $0.77(0.54-1.11)$ & 0.16 \\
\hline $\begin{array}{l}\text { OR }=\text { odds ratio. Hosmer-Lemeshow H statistic for goodness of fit, 5.31; } P=0.72 ; \text { area under } \\
\text { receiver-operator characteristic curve, } 0.80 .\end{array}$ &
\end{tabular}

both public and private spaces varies between individuals. Completing the survey led $35 \%$ of students to adjust their privacy settings, suggesting that being prompted to do so, combined with their reflecting on a desirable public image, may be an effective intervention. The higher proportions of participants who reported having seen rather than posted unprofessional behaviour also highlight an intrinsic attribute of social networks: that a single example of unprofessional content may be seen by a large number of medical students. While use of a private profile may not reduce the incidence of unprofessional content, it does reduce the size of the potential audience for that content.

\section{Strengths and limitations}

There are some limitations to our study. Participation was voluntary, and many participants were recruited through social media; each factor introduces selection bias. Our survey included only a small proportion of the 16993 Australian medical students in 2013; we were unable to estimate the number of students who were actually aware of the study. A large proportion of participants were from a small number of Victorian universities, the state in which the authors of this article reside, and this may limit extrapolation of the results to other medical student populations. The survey was conducted in 2013, and the time that has elapsed between collecting and publishing our data is also acknowledged.

Our study relied on the self-reporting of specific content on social media, and did not record the prevalence of unprofessional behaviour itself. This has the potential for introducing both information and recall bias, as students may report their own behaviour differently to their perception of others'. In addition, the accuracy of participants' responses could not be verified. The findings of this study cannot be extrapolated to qualified medical practitioners or to other allied medical staff.

Nevertheless, our study is the largest to examine medical student professionalism on social media, and has identified factors that may predict and protect against future unprofessional behaviour. It also showed that the act of completing the study was sufficient to change some behaviours, so that introspection itself may be a beneficial tool for educators seeking to address this problem.

\section{Conclusion}

The use of social media by the surveyed students was almost universal, and unprofessional behaviours on social media were exhibited by a significant proportion of medical students, despite widespread awareness of guidelines about professionalism. Content posted online is effectively in the public domain, and management of their online identity is therefore now part of a student's professional responsibility. Medical educators should consider approaches beyond simply providing guidelines or policies, and students should be regularly prompted to reflect on their activities, to evaluate their online behaviours, and to temper them if appropriate.

Competing interests: Stewart Morrison and Hugh Stephens were co-authors of the Australian Medical Association's Social Media and the Medical Profession guideline. Hugh Stephens is the Chief Executive Officer of Dialogue Consulting, a social and digital media strategy, risk management and advisory service, and has participated at conferences on social media in health care as a paid and non-paid speaker.

(c) 2015 AMPCo Pty Ltd. Produced with Elsevier B.V. All rights reserved. 
1 Kaplan A, Haenlein M. Users of the world, unite! The challenges and opportunities of Social Media. Bus Horizons 2010; 53: 59-68.

2 Thompson LA, Dawson K, Ferdig R, et al. The intersection of online social networking with medical professionalism. J Gen Intern Med 2008; 23: 954-957.

3 Usher K, Woods C, Casellac E, et al. Australian health professions student use of social media. Collegian 2014; 21: 95-101.

4 McCartney M. How much of a social media profile can doctors have? BMJ 2012; 344: e440.

5 Chretien KC, Greysen SR, Chretien JP, Kind T. Online posting of unprofessional content by medical students. JAMA 2009; 302: 1309-1315.
6 Essary AC. The impact of social media and technology on professionalism in medical education. J Physician Assist Educ 2011; 22: 50-53.

7 Mansfield SJ, Morrison SG, Stephens $\mathrm{HO}$, et al. Social media and the medical profession. Med J Aust 2011; 194: 642-644. https://www.mja.com.au/ journal/2011/194/12/social-media-andmedical-profession

8 British Medical Association. Using social media: practical and ethical guidance for doctors and medical students. London: BMA, 2011. http:// bma.org.uk/-/media/Files/PDFs/ Practical advice at work/Ethics/ socialmediaguidance.pdf (accessed Sep 2015).

9 Medical Deans Australia and New Zealand. Total medical student enrolments 2013 by year of course [website]. http://www.medicaldeans. org.au/wp-content/uploads/WebsiteStats-2013-Table-2.pdf (accessed May 2015).

10 Dyrbye LN, Massie FS, Eacker A, et al. Relationship between burnout and professional conduct and attitudes among US medical students. JAMA 2010; 304: 1173-1180.

1 Vartabedian BS, Amos E, Baruch J. Anonymous physician blogging. Virtual Mentor 2011; 13: 440-447.

12 Farnan JM, Paro JA, Higa JT, et al. The relationship status of digital media and professionalism: it's complicated. Acad Med 2009; 84: 1479-1481.

13 Cruess S, Cruess R. Professionalism must be taught. BMJ 1997; 315: 1674. 